\title{
A laboratory test based on determination of cytokine profiles: a promising assay to identify exposition to contact allergens and predict the clinical outcome in occupational allergic contact dermatitis
}

Valentina Bordignon ${ }^{1 *}$, Francesca Palamara², Giorgia Altomonte ${ }^{2}$, Isabella Sperduti ${ }^{3}$, Mario Pietravalle ${ }^{1}$, Claudia Cavallotti ${ }^{4}$, Paola Cordiali-Fei ${ }^{1}$, Maria Pia Fuggetta ${ }^{5}$, Antonio Cristaudo ${ }^{2}$ and Fabrizio Ensoli ${ }^{1}$

\begin{abstract}
Background: Para-phenylenediamine (PPD) is the main allergen causing adverse reactions to hair dyes and a frequent cause of occupational-related skin sensitization among hairdressers and beauticians. The immunologic mechanism of the disease relies on the production of inflammatory cytokines by allergen-specific T cells, while regulatory $T$ cells are thought to down-modulate the allergic response. This study was aimed at investigating the expression of effector or regulatory cytokines in exposed subjects in order to verify whether different cytokine profiles might predict distinct clinical outcomes. Peripheral blood mononuclear cells (PBMC) from 21 subjects occupationally exposed or not (10) to PPD were kept in short term cultures in the presence of optimized concentrations of NiSO $4 \times 6 \mathrm{H} 2 \mathrm{O}$ or PPD. The production of IFN- $\gamma$ and IL-10 elicited by antigens were analyzed by the ELISpot assay.

Results: The presence of IFN- $\gamma$ responses toward PPD was significantly correlated with a positive patch test $(P=0.002)$ and allergic symptoms, while IL10 responses were invariably found in PPD-exposed but clinically asymptomatic subjects with negative patch testing. We found concordance between the different cytokine profiles and patch test results. No false-positive results were found for the different cytokine profiles induced by PPD, resulting in 100\% specificity. The sensitivity of the test was $87.5 \%(95 \% \mathrm{Cl} 65.9-100.0)$ with an overall test accuracy of $93.3 \%$. Although larger prospective-retrospective studies are necessary to validate the predictive potential of the test, the negative and positive predicted values for PPD in this study were NPV $=87.5 \%$ and PPV $=100 \%$, respectively.

Conclusions: These data indicate that distinct cytokine profiles are associated with different clinical manifestations. The test, which is based on a simple and rapid profiling of cytokine responses by T lymphocytes against allergens, has proven to be a promising laboratory tool, useful for both the identification of previous contact with allergens and the etiologic diagnosis of contact allergies as well as capable of predicting the clinical outcome (development of an allergic or tolerant response).
\end{abstract}

Keywords: Allergic contact dermatitis, Cytokines, ELISpot, Nickel, Occupation, Para-phenylenediamine, Patch test

\footnotetext{
* Correspondence: bordignon@ifo.it

${ }^{1}$ Clinical Pathology and Microbiology, San Gallicano Dermatology Institute,

Via Elio Chianesi 53, Rome 00144, Italy

Full list of author information is available at the end of the article
} 


\section{Background}

Para-phenylenediamine (PPD) and related para-amino compounds represent the most common active reagents present in permanent dyes, which are widely used in a variety of industrial processes. PPD and related agents are potent contact allergens [1-5]. The relevance of PPD in the pathogenesis of delayed-type occupational skin allergy has increased considerably in recent years [6] and has been recognised as the main allergen causing severe adverse reactions to hair dyes $[7,8]$. In addition, PPD-related substances are also present in textile dyes [9], leather dyes [10], fur dyes, and 'black' rubbers [11]. Occupational sensitization to PPD and related substances presents the highest prevalence among hairdressers [12] and beauticians, in both men (15.4\%) and women (14.7\%) [5]. However, severe allergic reactions to PPD have been also reported in children [6]. In addition, the increasing use of permanent makeup or tattoos has recently become a further cause of PPD skin sensitization $[13,14]$.

The clinical outcome of allergic contact dermatitis (ACD) includes contact urticaria, lymphomatoid reactions and even anaphylaxis [15], causing a considerable social and economic burden [7].

PPD-induced allergic disorders are mostly caused by skin contact to low molecular weight haptens. In fact, the effects of PPD on immune cells is thought to arise from its chemical instability under aqueous conditions. The ensuing auto-oxidation of the molecule leads to the formation of an electrophilic primary quinonediimine intermediate, which is susceptible to sequential self-conjugation. A rearrangement product of the oxido-conjugation reaction gives rise to the trimeric Bandrowski's base, which is also immunogenic by itself $[16,17]$.

The process of hypersensitivity in ACD requires a first sensitization phase, in which the hapten-carrier complex leads to T-cell activation [18-20] and is followed by the local release of pro-inflammatory cytokines. This causes skin inflammation with the involvement of keratinocytes, which is followed by epidermal changes, including spongiosis and, macroscopically, to the development of scales, vesicles or pustules [21]. Thus, immunologic mechanism responsible for ACD rely on the production of inflammatory cytokines by allergen-specific T cells, while regulatory cytokines are thought to down-modulate the allergic response. Previous studies have shown a relationship between the profile of cytokines induced by $\mathrm{T}$ cells and the presence of a skin reactivity to PPD, suggesting that IL-10 production exerts a "protective" effect while IFN- $\gamma$ induces a "reactive" response [17,22,23]. These data support the hypothesis that a regulatory mechanism mediated by IL-10 contributes to the control of the clinical manifestations in response to allergens [24].

At present, diagnosis of ACD is based on clinical history and patch testing, the latter having major disadvantages, since interpretation of the results is subjective. Thus, the development of an accurate and reliable in vitro assay based on informative biomarkers and capable of predicting the clinical outcome, still represents an unmet need for the clinical and therapeutic management of ACD [25].

The present study was aimed at developing a laboratory test capable of exploring the cytokine profiles expressed by peripheral blood mononuclear cells (PBMC) in response to PPD and another common active allergen such as Nickel (Ni), and evaluating its specificity and sensitivity as well as the potential for predicting the clinical outcome in exposed subjects.

\section{Methods \\ Patients}

PBMC were obtained from 31 subjects. Twenty-one of them were hairdressers (13 women and 8 men). Eleven of them attended the Allergy outpatient clinic presenting moderate $(n=8)$ or severe $(n=3)$ symptoms of ACD, while 10 subjects were asymptomatic. Ten healthy subjects not occupationally exposed to PPD nor to Ni were studied as controls. The mean age was 33.2 yrs, with a range between 20-55. Subject's description is summarized in Table 1.

Patient assessment was based on the administration of a questionnaire to collect information including occupational history, personal and familiar history of allergy (asthma and/or allergic rhino-conjunctivitis with at least one positive prick test reaction to relevant aeroallergens), and dermatitis. None of the patients had recently used immunosuppressive medication or underwent UV radiation or suffered from acute inflammatory skin diseases. Further, none of them had metal dental braces neither the presence of tattoo.

An informed consent was obtained from all subjects prior to the blood samples collection (Ethics Committee approval N. 488/14 IFO, Istituti Fisioterapici Ospitalieri).

\section{Patch testing}

Patch testing was performed by Finn Chambers ${ }^{\odot}$ on Scanpor tape, with the European standard series of contact allergens (Hermal Trolab, Reinbeck, Germany), including 5\% Nickel Sulphate hexahydrate $\left(\mathrm{NiSO}_{4} \times 6 \mathrm{H}_{2} \mathrm{O}\right.$, Merck, AG, Darmstadt, F.R.G) and PPD 1\% (FIRMA, Firenze, Italy; Chemotechnique Diagnostics, Vellinge, Sweden) applied in petrolatum. All allergens were applied on the upper back and removed after $48 \mathrm{hr}$.

Patch test responses were examined on day 2 and defined as strong (+++: oedema, erythema, papules and vesicles), moderate (++: oedema, erythema and papules), weak (+: oedema and erythema) or no reaction (neg) according to the International Contact Dermatitis Research Group guidelines [26]. 
Table 1 Clinical details of patients

\begin{tabular}{|c|c|c|c|c|c|c|c|}
\hline Patients & Age & Sex & Occupationally exposed & ACD symptoms & Patch test PPD & Patch test $\mathrm{Ni}$ & Other Patch test positivities \\
\hline 1 & 25 & $f$ & Yes & Severe & +++ & ++ & parabens, colophony, disp yellow \\
\hline 13 & 26 & $f$ & Yes & Severe & +++ & ++ & No \\
\hline 2 & 25 & $f$ & Yes & Severe & ++ & neg & No \\
\hline 3 & 35 & $f$ & Yes & Moderate & ++ & neg & No \\
\hline 4 & 55 & f & Yes & Moderate & ++ & neg & No \\
\hline 5 & 20 & $\mathrm{~m}$ & Yes & Moderate & + & neg & No \\
\hline 14 & 31 & $\mathrm{~m}$ & Yes & Moderate & ++ & neg & No \\
\hline 15 & 40 & $f$ & Yes & Moderate & + & neg & No \\
\hline 10 & 25 & $f$ & Yes & Moderate & neg & + & No \\
\hline 11 & 29 & $f$ & Yes & Moderate & neg & ++ & No \\
\hline 12 & 26 & $f$ & Yes & Moderate & neg & + & No \\
\hline 6 & 31 & $f$ & Yes & Asymptomatic & neg & neg & No \\
\hline 7 & 41 & $f$ & Yes & Asymptomatic & neg & neg & No \\
\hline 8 & 43 & $f$ & Yes & Asymptomatic & neg & neg & katon \\
\hline 9 & 33 & $\mathrm{~m}$ & Yes & Asymptomatic & neg & neg & No \\
\hline 20 & 41 & $f$ & Yes & Asymptomatic & neg & neg & No \\
\hline 21 & 41 & $\mathrm{~m}$ & Yes & Asymptomatic & neg & neg & No \\
\hline 22 & 33 & $\mathrm{~m}$ & Yes & Asymptomatic & neg & neg & No \\
\hline 23 & 35 & $\mathrm{~m}$ & Yes & Asymptomatic & neg & neg & No \\
\hline 24 & 32 & $\mathrm{~m}$ & Yes & Asymptomatic & neg & neg & No \\
\hline 25 & 33 & $\mathrm{~m}$ & Yes & Asymptomatic & neg & neg & No \\
\hline 26 & 35 & $f$ & Not & Asymptomatic & neg & neg & No \\
\hline 27 & 41 & $f$ & Not & Asymptomatic & neg & neg & No \\
\hline 28 & 43 & $f$ & Not & Asymptomatic & neg & neg & No \\
\hline 29 & 38 & $f$ & Not & Asymptomatic & neg & neg & No \\
\hline 30 & 38 & $f$ & Not & Asymptomatic & neg & neg & No \\
\hline 31 & 37 & $f$ & Not & Asymptomatic & neg & neg & No \\
\hline 32 & 25 & $f$ & Not & Asymptomatic & neg & neg & No \\
\hline 33 & 23 & $\mathrm{~m}$ & Not & Asymptomatic & neg & neg & No \\
\hline 34 & 24 & $\mathrm{~m}$ & Not & Asymptomatic & neg & neg & No \\
\hline 35 & 25 & $\mathrm{~m}$ & Not & Asymptomatic & neg & neg & No \\
\hline
\end{tabular}

\section{PBMC isolation}

PBMC were isolated from $10 \mathrm{ml}$ of heparinized blood, collected 48 hrs after skin testing, by standard Ficoll density-gradient centrifugation (Lympholyte- $\mathrm{H}$ solution Cederlane, Ontario, Canada) and washed twice with PBS. Cell aliquots were frozen in 90\% heat inactivated Fetal Bovine Serum (FBS, Euroclone) and 10\% DMSO (Dimethylsulphoxide, Sigma) and kept in liquid nitrogen.

\section{ELISpot determination of cytokine profiles induced in PBMC in response to allergens}

Cytokine profiles were determined by Dual-Color Human ELISpot assay, according to manufacturer's instruction. This test allow the simultaneous detection of IFN- $\gamma$ and
IL-10 secreting cells (R\&D Systems, Europe Ltd., Abingdon, UK). Briefly, PBMC $\left(3 \times 10^{5}\right.$ cells/well) from patients with positive patch test to PPD or Ni or both, or with negative patch test responses to either antigen, were incubated in triplicate $\left(37^{\circ} \mathrm{C}, 5 \% \mathrm{CO}_{2}\right)$ for $48 \mathrm{~h}$, in the presence or in the absence of the allergens, in RPMI-1640 medium containing $50 \mathrm{IU} / \mathrm{mL}$ penicillin, $50 \mu \mathrm{g} / \mathrm{mL}$ streptomycin, $2 \mathrm{mmol} /$ L L-glutamine, $1 \times$ mixture of nonessential amino acids, $10 \%$ FCS (Gibco, BRL, UK). Red (IFN- $\gamma$ ) and blue (IL-10) spots generated by cells producing cytokines were assessed and recorded by an Automated ImmunoSpot Image Analyzer Software (AELVIS Technologies, TEMA ricerche, Italy).

The number of spot forming cells (SFC) per $3 \times 10^{5}$ PBMC was used for the calculation of the Stimulation 
Index (S.I.), which express the ratio between stimulated and unstimulated cells [24]. Responses were considered positive when S.I. $\geq 3$.

\section{Allergens}

The allergens used for in vitro stimulation of PBMC were: Nickel sulphate hexahydrate $\mathrm{NiSO}_{4} \times 6 \mathrm{H}_{2} \mathrm{O}$, (Merck AG, Darmstadt, F.R.G), PPD (Sigma-Aldrich, Chemie, Kappelweg, Schnelldorf, Germany). Triplicate wells containing unstimulated cells or mitogen stimulated cells (PHA $1 \mu \mathrm{g} /$ $\mathrm{ml}$, Sigma, Saint Louis, Missouri, USA) were the negative and positive controls, respectively.
Stock solutions of PPD (1 M) were prepared dissolving the commercial powder in cell culture medium/DMSO $(4: 1, \mathrm{v} / \mathrm{v})$ [27]. Preliminary titration experiments were performed with PPD on 5 different donors by testing different concentrations (0.1-1-10-20-40-50-100 $\mu \mathrm{M})$ of PPD. The results were consistent with previous studies [27] and showed that concentration over $50 \mu \mathrm{M}$ were toxic on PBMC (data not shown).

$\mathrm{NiSO}_{4}$ was resuspended before use, in sterile saline solution at $2 \mathrm{mg} / \mathrm{ml}$ (Bioindustria, Novi Ligure, Italy) and used at $20 \mu \mathrm{g} / \mathrm{ml}$ concentration as previously described [24].

Table 2 Individual values of IFN- $\gamma$ or IL-10 spot forming cells (expressed as mean values of triplicate wells) and S.I. for each experimental condition (W/O: medium alone; PHA: polyclonal stimulation $1 \mu \mathrm{g} / \mathrm{ml} ; \mathrm{Ni} 20 \mu \mathrm{g} / \mathrm{ml}, \mathrm{PPD} 10 \mu \mathrm{M}$ )

\begin{tabular}{|c|c|c|c|c|c|c|c|c|c|c|c|c|c|c|}
\hline \multirow[b]{2}{*}{ Patients } & \multicolumn{7}{|l|}{ IFN } & \multicolumn{7}{|l|}{ IL-10 } \\
\hline & $\mathrm{W} / 0$ & PHA & PHA S.I. & $\mathrm{Ni}$ & Ni S.I. & PPD & $\overline{\text { PPD S.I. }}$ & W/O & PHA & PHA S.I. & $\mathrm{Ni}$ & Ni S.I. & PPD & $\overline{\text { PPD S.I. }}$ \\
\hline 1 & 27.00 & 187.33 & 6.94 & 151.67 & 5.62 & 117.00 & 4.33 & 10.00 & 69.33 & 6.93 & 11.00 & 1.10 & 18.67 & 1.87 \\
\hline 13 & 17.67 & 120.33 & 6.81 & 42.00 & 2.38 & 188.67 & 10.68 & 18.33 & 96.67 & 5.27 & 13.33 & 0.73 & 15.33 & 0.84 \\
\hline 2 & 16.50 & 124.67 & 7.56 & 31.33 & 1.90 & 65.33 & 3.96 & 14.33 & 100.33 & 7.00 & 82.33 & 5.74 & 17.00 & 1.19 \\
\hline 3 & 27.67 & 285.00 & 10.30 & 38.00 & 1.37 & 143.00 & 5.17 & 13.00 & 92.00 & 7.08 & 62.33 & 4.79 & 17.33 & 1.33 \\
\hline 4 & 45.00 & 360.33 & 8.01 & 41.00 & 0.91 & 183.33 & 4.07 & 16.83 & 101.00 & 6.00 & 71.67 & 4.26 & 17.00 & 1.01 \\
\hline 5 & 35.50 & 285.00 & 8.03 & 30.00 & 0.85 & 127.67 & 3.60 & 9.33 & 73.67 & 7.89 & 37.67 & 4.04 & 10.67 & 1.14 \\
\hline 14 & 15.67 & 102.33 & 6.53 & 31.67 & 2.02 & 79.33 & 5.06 & 19.00 & 71.67 & 3.77 & 39.33 & 2.07 & 15.00 & 0.79 \\
\hline 15 & 20.67 & 91.33 & 4.42 & 18.67 & 0.90 & 37.33 & 1.81 & 10.33 & 58.67 & 5.68 & 48.33 & 4.68 & 12.67 & 1.23 \\
\hline 10 & 14.67 & 197.33 & 13.45 & 61.67 & 4.20 & 27.33 & 1.86 & 30.67 & 244.00 & 7.96 & 103.33 & 3.37 & 153.33 & 5.00 \\
\hline 11 & 22.00 & 155.33 & 7.06 & 84.33 & 3.83 & 25.67 & 1.17 & 17.67 & 75.33 & 4.26 & 18.33 & 1.04 & 62.33 & 3.53 \\
\hline 12 & 23.51 & 160.67 & 6.83 & 95.33 & 4.05 & 47.67 & 2.03 & 17.00 & 92.00 & 5.41 & 22.50 & 1.32 & 63.00 & 3.71 \\
\hline 6 & 12.00 & 92.00 & 7.67 & 13.67 & 1.14 & 5.67 & 0.47 & 6.50 & 53.00 & 8.15 & 47.33 & 7.28 & 34.00 & 5.23 \\
\hline 7 & 23.33 & 190.00 & 8.14 & 22.00 & 0.94 & 9.67 & 0.41 & 15.67 & 117.33 & 7.49 & 84.00 & 5.36 & 72.67 & 4.64 \\
\hline 8 & 21.67 & 198.33 & 9.15 & 15.33 & 0.71 & 57.00 & 2.63 & 6.67 & 52.00 & 7.80 & 32.00 & 4.80 & 11.33 & 1.70 \\
\hline 9 & 10.33 & 70.33 & 6.81 & 9.67 & 0.94 & 16.33 & 1.58 & 32.67 & 273.00 & 8.36 & 133.67 & 4.09 & 170.33 & 5.21 \\
\hline 20 & 17.00 & 72.33 & 4.25 & 18.67 & 1.10 & 10.67 & 0.63 & 4.50 & 57.00 & 12.67 & 42.67 & 9.48 & 37.33 & 8.30 \\
\hline 21 & 7.33 & 204.67 & 27.91 & 8.33 & 1.14 & 15.00 & 2.05 & 16.00 & 104.67 & 6.54 & 88.33 & 5.52 & 76.00 & 4.75 \\
\hline 22 & 26.00 & 195.67 & 7.53 & 35.33 & 1.36 & 59.67 & 2.29 & 10.33 & 47.00 & 4.55 & 37.33 & 3.61 & 32.33 & 3.13 \\
\hline 23 & 14.00 & 72.00 & 5.14 & 12.00 & 0.86 & 18.33 & 1.31 & 31.67 & 277.00 & 8.75 & 139.33 & 4.40 & 173.67 & 5.48 \\
\hline 24 & 10.00 & 97.00 & 9.70 & 17.00 & 1.70 & 8.33 & 0.83 & 9.83 & 54.00 & 5.49 & 50.67 & 5.15 & 36.00 & 3.66 \\
\hline 25 & 17.67 & 207.33 & 11.74 & 26.67 & 1.51 & 13.00 & 0.74 & 19.33 & 121.00 & 6.26 & 86.00 & 4.45 & 76.00 & 3.93 \\
\hline 26 & 18.33 & 208.67 & 11.38 & 18.67 & 1.02 & 27.33 & 1.49 & 7.00 & 57.33 & 8.19 & 16.67 & 2.38 & 11.33 & 1.62 \\
\hline 27 & 3.67 & 70.33 & 19.18 & 9.67 & 2.64 & 9.00 & 2.45 & 32.67 & 273.00 & 8.36 & 33.67 & 1.03 & 70.33 & 2.15 \\
\hline 28 & 21.67 & 228.67 & 10.55 & 22.00 & 1.02 & 30.67 & 1.42 & 4.00 & 56.67 & 14.17 & 11.67 & 2.92 & 9.33 & 2.33 \\
\hline 29 & 32.67 & 68.33 & 2.09 & 13.00 & 0.40 & 16.00 & 0.49 & 3.67 & 43.67 & 11.91 & 9.67 & 2.64 & 9.00 & 2.45 \\
\hline 30 & 20.00 & 206.00 & 10.30 & 19.33 & 0.97 & 33.33 & 1.67 & 9.67 & 52.00 & 5.38 & 28.00 & 2.90 & 14.67 & 1.52 \\
\hline 31 & 25.00 & 66.33 & 2.65 & 26.33 & 1.05 & 23.00 & 0.92 & 32.67 & 173.00 & 5.30 & 32.00 & 0.98 & 66.00 & 2.02 \\
\hline 32 & 6.00 & 36.00 & 6.00 & 15.33 & 2.56 & 17.67 & 2.94 & 6.67 & 52.00 & 7.80 & 15.33 & 2.30 & 11.33 & 1.70 \\
\hline 33 & 9.33 & 51.00 & 5.46 & 9.67 & 1.04 & 16.33 & 1.75 & 28.33 & 212.33 & 7.49 & 50.00 & 1.76 & 62.67 & 2.21 \\
\hline 34 & 32.00 & 208.67 & 6.52 & 19.33 & 0.60 & 29.00 & 0.91 & 7.33 & 53.00 & 7.23 & 18.67 & 2.55 & 12.00 & 1.64 \\
\hline 35 & 5.33 & 70.33 & 13.19 & 6.33 & 1.19 & 14.33 & 2.69 & 21.67 & 273.00 & 12.60 & 26.67 & 1.23 & 27.00 & 1.25 \\
\hline
\end{tabular}


All stock solutions were tested to exclude LPS contamination (Limulus assay, BioWhittaker, Cambrex Company, USA).

\section{Statistical analysis}

Descriptive statistic was used to summarize pertinent study information. Comparisons between groups were performed for different variables using the non parametric Mann-Whitney test. Agreement between the in vitro results and clinical patch testing was estimated using the Cohen's kappa test. Specificity, sensitivity, negative and positive predicted value (NPV and PPV, respectively) and accuracy were calculated. Significance was assessed at $5 \%$ level. All analyses were performed by SPSS for Windows statistical software (version 20; SPSS Inc., Chicago IL, USA).

\section{Results}

Patch testing

Patch test was performed in all 31 subjects with or without an history of occupational contact with PPD. Subject's description and patch test results are detailed in Table 1. According to the skin response, 8 subjects out of 31 presented a skin response to PPD, while 5 subjects had responses to Ni. Two subjects (Id: 1 and 13, respectively) had positive responses to both allergens. All 20 asymptomatic subjects had negative patch test to PPD and $\mathrm{Ni}$.

\section{Cytokine profiles in response to PPD and $\mathrm{Ni}$}

PBMC were incubated with PPD or $\mathrm{Ni}$ in short term cultures and IFN- $\gamma$ and IL-10 production was measured by ELISpot assay. The results including SFC and S.I. for each subject are shown in Table 2. In all cases PHA stimulation (positive control) excluded individual differences in the capability of producing a specific cytokine as well as differences due to the quality of the cryopreservation.

The production of IFN- $\gamma$ was strictly associated with a positive patch test result (Table 3 ). In fact, Positive IFN- $\gamma$-responses (Index $\geq 3$ ), in the presence of PPD or $\mathrm{Ni}$, were detected only in subjects with positive patch test to PPD (Figure 1, panel A, P=0.002) or to Ni (Figure 1, panel $\mathrm{C}, \mathrm{P}=0.001)$, respectively.

All asymptomatic subjects with a history of contact with PPD, however presenting a negative patch test, had invariably an IL-10 response in the absence of IFN- $\gamma$ production. Healthy controls did not produce IFN- $\gamma$ nor IL-10 in response to stimulation with PPD or Ni.

As shown in Figure 1, IFN- $\gamma$ or IL-10 production in response to allergens appear mutually exclusive, while PHA is capable of inducing both cytokines (Table 2). Statistical analysis confirmed a significant negative correlation between IFN- $\gamma$ and IL-10 production $(\mathrm{P}<0.0001)$.
Table 3 PPD or Ni patch test results and respective S.I. IFN- $\gamma$ values in response to antigens

\begin{tabular}{|c|c|c|c|c|}
\hline Patients & Patch test PPD & SI PPD & Patch test Ni & SI ni \\
\hline 1 & +++ & 4.33 & ++ & 5.62 \\
\hline 13 & +++ & 10.68 & ++ & 2.38 \\
\hline 2 & ++ & 3.96 & neg & 1.90 \\
\hline 3 & ++ & 5.17 & neg & 1.37 \\
\hline 4 & ++ & 4.07 & neg & 0.91 \\
\hline 5 & + & 3.60 & neg & 0.85 \\
\hline 14 & ++ & 5.06 & neg & 2.02 \\
\hline 15 & + & 1.81 & neg & 0.90 \\
\hline 10 & neg & 1.86 & + & 4.20 \\
\hline 11 & neg & 1.17 & ++ & 3.83 \\
\hline 12 & neg & 2.03 & + & 4.05 \\
\hline 6 & neg & 0.47 & neg & 1.14 \\
\hline 7 & neg & 0.41 & neg & 0.94 \\
\hline 8 & neg & 2.63 & neg & 0.71 \\
\hline 9 & neg & 1.58 & neg & 0.94 \\
\hline 20 & neg & 0.63 & neg & 1.10 \\
\hline 21 & neg & 2.05 & neg & 1.14 \\
\hline 22 & neg & 2.29 & neg & 1.36 \\
\hline 23 & neg & 1.31 & neg & 0.86 \\
\hline 24 & neg & 0.83 & neg & 1.70 \\
\hline 25 & neg & 0.74 & neg & 1.51 \\
\hline 26 & neg & 1.49 & neg & 1.02 \\
\hline 27 & neg & 2.45 & neg & 2.64 \\
\hline 28 & neg & 1.42 & neg & 1.02 \\
\hline 29 & neg & 0.49 & neg & 0.40 \\
\hline 30 & neg & 1.67 & neg & 0.97 \\
\hline 31 & neg & 0.92 & neg & 1.05 \\
\hline 32 & neg & 2.94 & neg & 2.56 \\
\hline 33 & neg & 1.75 & neg & 1.04 \\
\hline 34 & neg & 0.91 & neg & 0.60 \\
\hline 35 & neg & 2.69 & neg & 1.19 \\
\hline
\end{tabular}

No false-positive test results were found for PPD- neither for $\mathrm{Ni}$-induced increments, thus giving a 100\% specificity for both allergens. On the other hand, the sensitivity was calculated as $87.5 \%$ (95\% CI 65.9-100.0) for PPD and 80\% (95\% CI 57.4-100.0) for Ni, respectively, hence the overall test accuracy was of $93.3 \%$.

A satisfactory concordance agreement between in vitro and patch test results was also found (Cohen's kappa test 0.84, $\mathrm{P}=0.001$ ).

The negative and positive predicted values were NPV = $87.5 \%$ and PPV $=100 \%$ for PPD and $\mathrm{NPV}=90.9 \%$ and $\mathrm{PPV}=100 \%$, for $\mathrm{Ni}$, respectively. 
A

IFN- $\gamma$

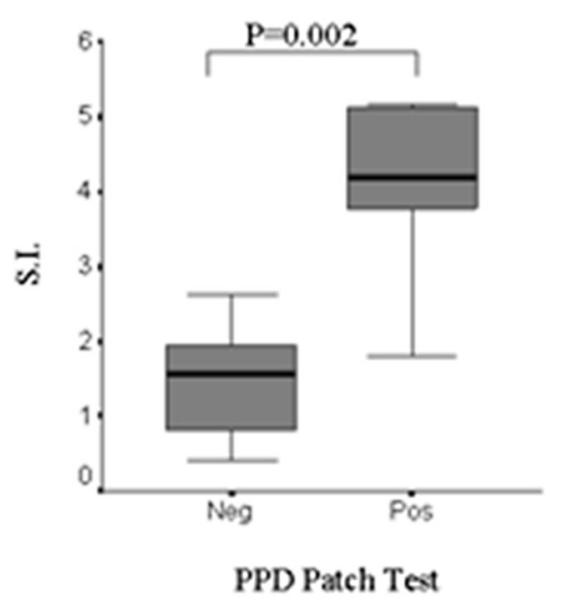

C

IFN- $\gamma$

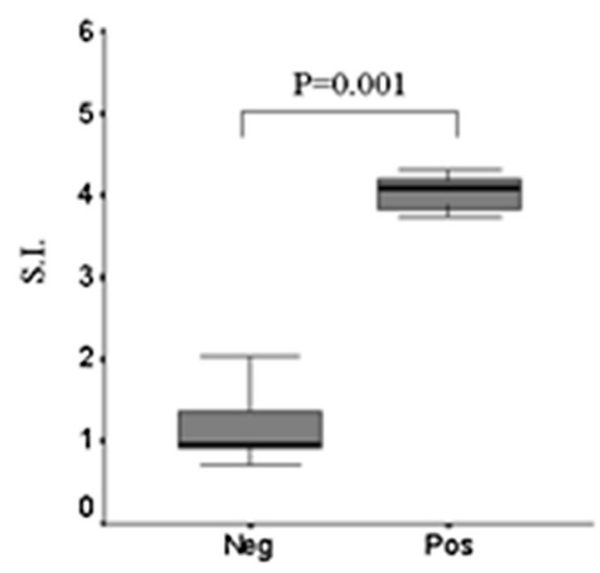

Ni Patch Test
B $\mathrm{IL}-10$

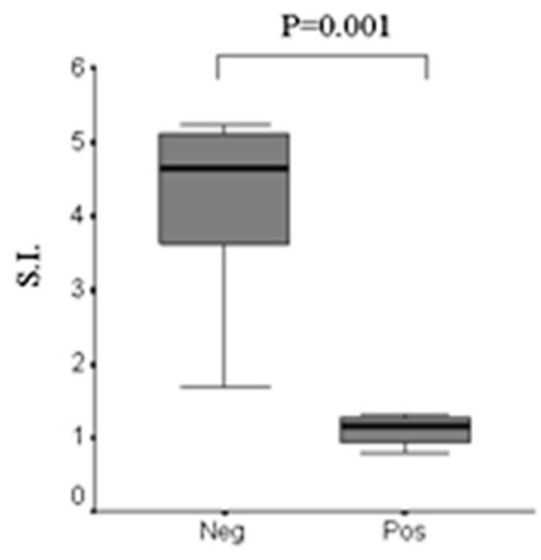

PPD Patch Test

D $\mathrm{IL}-10$

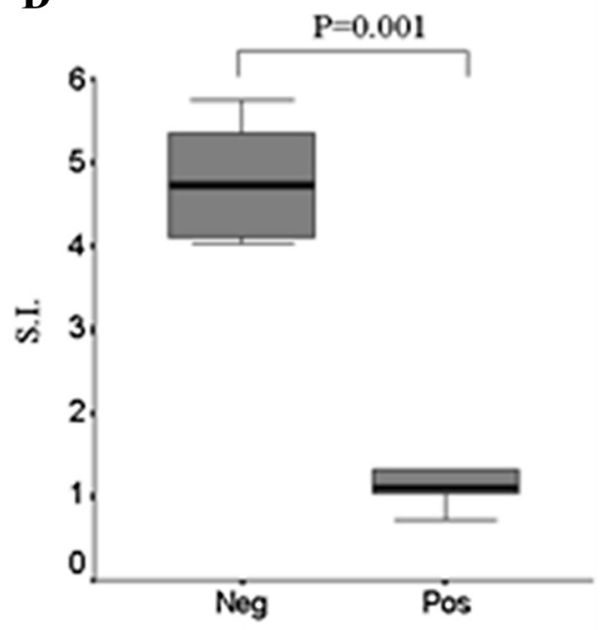

Ni Patch Test

Figure 1 Cytokine response elicited by PPD (panel A and B) or Ni (panel C and D) by ELISpot analysis in patch test negative or positive patients, respectively. ELISpot results of detection of IFN- (panel A-C) or IL-10 (panel B-D) producing PBMC upon stimulation with Ni (20 $\mu \mathrm{g} / \mathrm{ml}$ ) or PPD $(10 \mu \mathrm{M})$ were expressed as the mean values of S.I. \pm SD. S.I. Index value is expressed by the ratio between the number of spot forming cells (IFN- $\gamma$ and IL-10 producing PBMC) upon stimulation with the allergen and those present in the absence of stimuli (spontaneous cytokine production). Positive IFN- - -responses (Index $\geq 3$ ), in the presence of PPD or Ni, were detected only in subjects with positive patch test to PPD (panel $\mathbf{A}, \mathrm{P}=0.002$ ) or to $\mathrm{Ni}$ (panel $\mathbf{C}, \mathrm{P}=0.001$ ), respectively.

\section{Discussion}

This study was aimed at exploring the cytokine responses to specific allergens to evaluate whether the analysis of different cytokine profiles might provide the basis for a laboratory test capable of identify an allergic sensitization.

To date, the patch test is considered the gold standard for identifying the causative agents responsible for contact allergy [28]. However, its use is currently debated $[4,29]$. In fact, although having a high sensitivity, patch test has a major disadvantage, since interpretation of the results is subjective. In addition it might be the cause of a iatrogenic sensitization and, although rarely, induce adverse reactions [30]. Thought desirable, a laboratory test capable of supporting the clinical and therapeutic management of ACD it is not available, as yet.

Evidence indicate that the clinical manifestations of $\mathrm{ACD}$ are associated with an inflammatory response to allergens [22] while "regulatory" responses [21] are found in allergen-responsive subject, which however do not develop clinical symptoms. In fact, previous studies [24,31,32] suggested that the production of specific cytokines in response to antigenic stimulation can effectively modulate the type of immune response. In particular, this paradigma strongly suggests that IFN- $\gamma$ production is associated with 
a "reactive" phenotype, which gives rise to clinical symptoms, while IL-10 production exerts a "protective" effect, capable of controlling the hypersensitivity symptoms in response to allergens [33]. This notion prompted us at exploring the pattern of cytokine expression elicited "in vitro" by contact allergens for the development of a novel, simple, robust and reliable laboratory test for the diagnosis and clinical profiling of ACD patients.

Other laboratory test, including the lymphocyte activation test (LAT) and lymphocyte transformation test (LTT), have been proposed to investigate drug and contact allergy [34-37]. These test are based on the detection of antigen-driven $\mathrm{T}$ cell proliferation by $\left[{ }^{3} \mathrm{H}\right]$-thymidine incorporation. However these approaches have shown a low reproducibility, being therefore difficult to standardize, and require radioactive compounds. Furthermore they do not provide any predictive information about the potential clinical outcome.

The present study was based on the assessment of the cytokine profiles, namely IFN- $\gamma$ and IL-10 production by $\mathrm{T}$ cells, in response to allergens, using a Dual-Color ELISpot assay [38,39].

We found a significant correlation between the ELISpot results and the results gathered by patch testing $(\mathrm{P}=0.001)$. In particular, IFN- $\gamma$ responses against PPD or $\mathrm{Ni}$ were found only in subjects with a positive patch test to PPD $(\mathrm{P}=0.002)$ or to $\mathrm{Ni}(\mathrm{P}=0.001)$, respectively. On the contrary, all clinically asymptomatic subjects, with a history of regular contact with PPD, had a negative patch test but showed an IL-10 production in response to the allergens, invariably in the absence of IFN- $\gamma$ production, while, control healthy subjects did not produce IFN- $\gamma$ nor IL-10. These data confirm the presence of an anamnestic, though clinically asymptomatic, response to the allergen even in exposed subject which present with a negative patch test. In fact, IFN- $\gamma$ or IL-10 production appear mutually exclusive, as confirmed by the statistical treatment of the data. The assay gave a $100 \%$ specificity, a $80-87,5 \%$ sensitivity and a $93.3 \%$ accuracy.

\section{Conclusions}

Although larger prospective-retrospective studies are necessary to validate the predictive potential of the test and the possible indications for its use in clinical practice, the results suggest that this assay might offer a complementary or, in some cases, alternative diagnostic tool for the assessment of individuals with known or suspected exposure to cutaneous allergens, particularly in those cases of difficult application of patch test as in patients with chronic skin inflammation, while helping avoid the risk of both subjective interpretation and iatrogen sensibilization. In addition, it may be useful to reveal previous exposure to allergens both in asymptomatic and "allergic" individuals and might prove effective at predicting the clinical outcome as well as at monitoring the effect of therapeutic regimens.

\section{Abbreviations}

PPD: Para-phenylenediamine; Ni: Nickel; PBMC: Peripheral blood mononuclear cells; SFC: Spot forming cells; S.I.: Stimulation index; LAT: Lymphocyte activation test; LTT: Lymphocyte transformation test.

\section{Competing interests}

The authors declare that they have no competing interests.

\section{Authors' contributions}

All authors of this paper have contributed to the study design and conduction. VB, PCF and FE conceived of the study design and manuscript preparation. VB performed the laboratory assessment and manuscript drafting. MPF provided the purified antigens for the in vitro testing. AC, MP, GA, CC and FP, performed the clinical studies and routine laboratory investigations. IS performed the statistical analyses. All authors read and approved the final manuscript.

\section{Acknowledgements}

This work was partially supported by Ministry of Health "Ricerca Corrente ISG 2011CdC 212140".

\section{Author details}

${ }^{1}$ Clinical Pathology and Microbiology, San Gallicano Dermatology Institute, Via Elio Chianesi 53, Rome 00144, Italy. ${ }^{2}$ Allergy and Infectious Diseases, San Gallicano Dermatology Institute, Via Elio Chianesi 53, Rome 00144, Italy. ${ }^{3}$ Epidermiology, Regina Elena Cancer Institute, Via Elio Chianesi 53, Rome 00144, Italy. ${ }^{4}$ Clinical Dermatology, San Gallicano Dermatology Institute, Via Elio Chianesi 53, Rome 00144, Italy. ${ }^{5}$ Institute of Translational Pharmacology (IFT), National Research Council (CNR), Rome, Italy.

Received: 10 July 2014 Accepted: 7 January 2015

Published online: 06 February 2015

\section{References}

1. Garrigue JL, Nicolas JF, Fraginals R, Benezra C, Bour H, Schmitt D. Optimization of the mouse ear swelling test for in vivo and in vitro studies of weak contact sensitizers. Contact Dermatitis. 1994;30:231-7.

2. Gerberick GF, Ryan CA, Dearman RJ, Kimber I. Local lymph node assay (LLNA) for detection of sensitization capacity of chemicals. Methods. 2007;41:54-60.

3. Xie Z, Hayakawa R, Sugiura M, Kojima H, Konishi H, Ichihara G, et al. Experimental study on skin sensitization potencies and cross-reactivities of hair-dye-related chemicals in guinea pigs. Contact Dermatitis. 2000;42:270-5.

4. Sosted H, Menne T, Johansen JD. Patch test dose-response study of p-phenylenediamine: thresholds and anatomical regional differences. Contact Dermatitis. 2006;54:145-9.

5. Malvestio A, Bovenzi M, Hoteit M, Belloni Fortina A, Peserico A, Corradin MT, et al. p-Phenylenediamine sensitization and occupation. Contact Dermatitis. 2011;64:37-42.

6. DeLeo VA. P-Phenylenediamine. Dermatitis. 2006;17:53-5.

7. Sosted H, Hesse U, Menn'e T, Andersen KE, Johanson JD. Contact dermatitis to hair dyes in an adult Danish population - an interview based study. $\mathrm{Br}$ J Dermatol. 2005;153:132-5.

8. Laing ME, Powell FC, O'Sullivan D. The influence of contact dermatitis on career change in hairdressers. Contact Dermatitis. 2006;54:218-9.

9. Ryberg K, Goossens A, Isaksson M, Ryberg K, Goossens A, Isaksson M, et al. Is contact allergy to disperse dyes and related substances associated with textile dermatitis? Br J Dermatol. 2009;160:107-15.

10. Nardelli A, Taveirne M, Drieghe J, Carbonez A, Degreef H, Goossens A. The relation between the localization of foot dermatitis and the causative allergens in shoes: a 13-year retrospective study. Contact Dermatitis. 2005;53:201-6.

11. Malinauskiene L, Zimerson E, Bruze M, Ryberg K, Isaksson M. Patch testing with the textile dyes disperse orange 1 and disperse yellow 3 and some of their potential metabolites, and simultaneous reactions to para-amino compounds. Contact Dermatitis. 2012;67:130-40. 
12. Valks R, Conde-Salazar L, Malfeito J, Ledo S. Contact dermatitis in hairdressers, 10 years later: patch-test results in 300 hairdressers (1994 to 2003) and comparison with previous study. Dermatitis. 2005;16:28-31.

13. De Cuyper C. Permanent makeup: indications and complications. Clin Dermatol. 2008;26:30-4.

14. Kluger N. Cutaneous complications related to permanent decorative tattooing. Expert Rev Clin Immunol. 2010;6:363-71.

15. Patel S, Basketter DA, Jefferies D, White IR, Rycroft RJ, McFadden JP, et al Patch test frequency to $\mathrm{p}$-phenylenediamine: follow up over the last 6 years. Contact Dermatitis. 2007:56:35-7.

16. Picardo M, Cannistraci C, Cristaudo A, De Luca C, Santucci B. Study on cross-reactivity to the para group. Dermatologica. 1990;181:104-8.

17. Coulter EM, Jenkinson C, Farrell J, Lavergne SN, Pease C, White A, et al. Measurement of CD4+ and CD8+ T-lymphocyte cytokine secretion and gene expression changes in p-phenylenediamine allergic patients and tolerant individuals. J Invest Dermatol. 2010;130:161-74.

18. Mori T, Kabashima K, Yoshiki R, Sugita K, Shiraishi N, Onoue A, et al. Cutaneous hypersensitivities to hapten are controlled by IFN-g-upregulated keratinocyte Th1 chemokines and IFN-g-downregulated Langerhans cell Th2 chemokines. J Invest Dermatol. 2008;128:1719-27.

19. Gamerdinger K, Moulon C, Karp DR, Van Bergen J, Koning F, Wild D, et al. A new type of metal recognition by human $T$ cells: contact residues for peptide-independent bridging of $\mathrm{T}$ cell receptor and major histocompatibility complex by nickel. J Exp Med. 2003;197:1345-53.

20. Sieben S, Kawakubo Y, Al Masaoudi T, Merk HF, Blömeke B. Delayed-type hypersensitivity reaction to paraphenylenediamine is mediated by 2 different pathways of antigen recognition by specific alphabeta human T-cell clones. J Allergy Clin Immunol. 2002;109:1005-11.

21. Akiba H, Kehren J, Ducluzeau MT, Krasteva M, Horand F, Kaiserlian D, et al. Skin inflammation during contact hypersensitivity is mediated by early recruitment of $\mathrm{CD} 8+\mathrm{T}$ cytotoxic 1 cells inducing keratinocyte apoptosis. J Immunol. 2002;168:3079-87.

22. Cavani A, Nasorri F, Ottaviani C, Sebastiani S, De Pita O, Girolomoni G. Human CD25+ regulatory T cells maintain immune tolerance to nickel in healthy, nonallergic individuals. J Immunol. 2003;171:5760-8.

23. Cavani A, Nasorri F, Prezzi C, Sebastiani S, Albanesi C, Girolomoni G. Human $\mathrm{CD} 4+\mathrm{T}$ lymphocytes with remarkable regulatory functions on dendritic cells and nickel-specific Th1 immune responses. J Invest Dermatol. 2000;114:295-302.

24. Bordignon V, Palamara F, Cordiali-Fei P, Vento A, Aiello A, Picardo M, et al. Nickel, palladium and rhodium induced IFN-gamma and IL-10 production as assessed by in vitro ELISpot-analysis in contact dermatitis patients. BMC Immunol. 2008;9:19.

25. Traidl-Hoffmann C, Ring J. Is there an in vitro test for type IV allergy discriminating between sensitization and allergic disease? Clin Exp Allergy. 2008;38:1412-5.

26. Rietshel RL, Fowler Jr JF. Practical aspects of patch testing. In: Rietshel RL, Fowler Jr JF, editors. Fisher's Contact Dermatitis. 5th ed. Philadelphia: Lipincott Williams and Wilkins; 2001. p. 9-26.

27. Coulter EM, Farrell J, Mathews KL, Maggs JL, Pease CK, Lockley DJ, et al. Activation of human dendritic cells by p-phenylenediamine. J Pharmacol Exp Ther. 2007;320:885-92.

28. Devos SA, Van Der Valk PG. Epicutaneous patch testing. Eur J Dermatol. 2002;12:506-13.

29. Thyssen JP, Menné T, Nielsen NH, Linneberg A. Is there a risk of active sensitization to PPD by patch testing the general population? Contact Dermatitis. 2007:57:133-4.

30. Jung P, Sesztak-Greinecker G, Wantke F, Götz M, Jarisch R, Hemmer W. A painful experience: black henna tattoo causing severe, bullous contact dermatitis. Contact Dermatitis. 2006;54:219-20.

31. Borg L, Christensen JM, Kristiansen J, Nielsen NH, Menné T, Poulsen LK. Nickel-induced cytokine production from mononuclear cells in nickel-sensitive individuals and controls. Cytokine profiles in nickel-sensitive individuals with nickel allergy-related hand eczema before and after nickel challenge. Arch Dermatol Res. 2000;292:285-91.

32. Büdinger L, Hertl M. Immunologic mechanisms in hypersensitivity reactions to metal ions: an overview. Allergy. 2000;55:108-15.

33. Rustemeyer $T$, von Blomberg BM, van Hoogstraten IM, Bruynzeel DP, Scheper RJ. Analysis of effector and regulatory immune reactivity to nickel. Clin Exp Allergy. 2004;34:1458-66.
34. Denman EJ, Denman AM. The lymphocyte transformation test and gold hypersensitivity. Ann Rheum Dis. 1968;27:582-9.

35. Thomas P, Bandl WD, Maier S, Summer B, Przybilla B. Hypersensitivity to titanium osteosynthesis with impaired fracture healing, eczema, and T-cell hyperresponsiveness in vitro: case report and review of the literature. Contact Dermatitis. 2006;55:199-202.

36. Kneilling M, Caroli U, Grimmel C, Fischer J, Eichner M, Wieder T, et al. Para-phenylenediamine-specific lymphocyte activation test: a sensitive in vitro assay to detect para-phenylenediamine sensitization in patients with severe allergic reactions. Exp Dermatol. 2010;19:435-41.

37. Coulter EM, Jenkinson C, Wu Y, Farrell J, Foster B, Smith A, et al. Activation of T-cells from allergic patients and volunteers by $\mathrm{p}$-phenylenediamine and Bandrowski's base. J Invest Dermatol. 2008;128:897-905.

38. Jakobson E, Masjedi K, Ahlborg N, Lundeberg L, Karlberg AT, Scheynius A. Cytokine production in nickel-sensitized individuals analysed with enzyme-linked immunospot assay: possible implication for diagnosis. Br J Dermatol. 2002;147:442-9.

39. Lindemann M, Böhmer J, Zabel M, Grosse-Wilde H. ELISpot: a new tool for the detection of nickel sensitization. Clin Exp Allergy. 2003;33:992-8.

\section{Submit your next manuscript to BioMed Central and take full advantage of:}

- Convenient online submission

- Thorough peer review

- No space constraints or color figure charges

- Immediate publication on acceptance

- Inclusion in PubMed, CAS, Scopus and Google Scholar

- Research which is freely available for redistribution 